Wheater, D. M. (1955). J. gen. Microbiol. 12, 123-132.

\title{
The Gharacteristics of Lactobacillus acidophilus and Lactobacillus bulgaricus
}

\author{
By DOROTHY M. WHEATER* \\ National Institute for Research in Dairying, University of Reading
}

SUMMARY : A study of 94 strains of lactobacilli was made. These were divided into three groups by 'sugar' fermentations and tolerance of sodium chloride and sodium tauroglycocholate. Strains of Lactobacillus acidophilus fermented amygdalin, cellobiose, salicin and sucrose, were variable in their action on dextrin and maltose, but grew in $2 \%$ sodium chloride or sodium tauroglycocholate. Strains of L. bulgaricus fermented none of these sugars and failed to grow in $2 \%$ sodium chloride or in $2 \%$ sodium tauroglycocholate. A third intermediate group failed to ferment amygdalin, cellobiose, salicin or sucrose, but all fermented dextrin and maltose and grew in $2 \%$ sodium chloride. They failed to grow in $2 \%$ sodium tauroglycocholate.

From the early days of bacteriology, there has been difficulty in differentiating between Lactobacillus acidophilus and L. bulgaricus and there has, therefore, been some confusion between these organisms. In 1910 Metchnikoff suggested that $L$. bulgaricus could become established in the gut, but other findings, reviewed by Rettger \& Cheplin (1921), suggested that only $L$. acidophilus could be implanted. The controversy which then arose was probably due to the lack of tests which would identify these organisms.

Several authors have suggested methods of differentiating between Lactobacillus acidophilus and $\boldsymbol{L}$. bulgaricus, and some of these rely on a single characteristic. Sugar fermentations were used by some workers, including Rahe (1918) who found fermentation of lactose, sucrose and raffinose variable. He therefore suggested that they should be identified on their ability to ferment maltose. Kulp \& Rettger (1924) studied a number of strains of these organisms and concluded that the reactions of the two species were very similar, the differences being in degree rather than in kind. They found that maltose, sucrose and laevulose were fermented by $L$. acidophilus, but rarely by $L$. bulgaricus. This was confirmed later by Sherman \& Hodge (1940). Other workers who used sugar fermentations included Curran, Rogers \& Whittier (1933), but their results were very different from those of Kulp \& Rettger (1924). These conflicting results threw doubt on the value of these tests for identification, although it is probable that the variation was due to the inadequacies of, and differences in, the media used rather than to the fermentative variability of the organisms. Orla-Jensen (1919), for instance, found difficulty in reaching any conclusions in the fermentation tests he made, because his medium failed to support good growth. A further probable reason for some discrepancies is the attempt to fit the results to the names previously assigned to the organisms in some earlier work.

Sherman \& Hodge (1940) found that Lactobacillus acidophilus would initiate

* Present address: Aplin \& Barrett I.td., Yeovil, Somerset. 
growth at $\mathrm{pH} \mathbf{7 \cdot 5}$, whereas $L$. bulgaricus would not, but 'weak' strains of L. acidophilus behaved like L. bulgaricus. They also found growth temperatures to be unreliable. Tests for growth in $2.5 \%$ sodium chloride, for the quantity of carbon dioxide produced, and for ability to grow in a simple medium, gave more precise results.

The most recent study of Lactobacillus acidophilus was that of Rogosa, Wiseman, Mitchell, Disraely \& Beaman (1953), who described its characteristics and on the basis of fermentations of single sugars recognized four variant types. Their work, however, was confined to oral strains and was not concerned with $L$. bulgaricus.

Briggs (1953b) was one of the few who attempted to classify the genus Lactobacillus as a whole; she divided these organisms into eight main groups by physiological tests (Table 1). Most strains labelled Lactobacillus acidophilus and $L$. bulgaricus came into either group I, II or III, and these groups were divided from the remaining homofermentative strains either by their inability to grow at $15^{\circ}$ or $48^{\circ}$, or in $4 \%$ sodium chloride, or by their low resistance to heat. The three groups were separated from each other mainly by their maximum growth temperatures, and both groups I and II contained named strains of $L$. acidophilus and $L$. bulgaricus, whilst group III contained a few strains of the latter. Amongst the unclassified organisms in section I there were further strains of $L$. bulgaricus. It was concluded that further tests were required to confirm these groupings.

The descriptions of Lactobacillus acidophilus and $L$. bulgaricus given in Bergey's Manual (Breed, Murray \& Hitchens, 1948) do not embody details which permit the clear identification of the organisms. The sugars used give variable results, and the 'distinctive characters' do not admit of easy routine differentiation. The aim of the present work was to define $L$. acidophilus and L. bulgaricus more clearly and to add, by way of simple tests, to the few known differential characteristics.

\section{METHODS}

Cultures and maintenance. The 94 cultures studied in this work were those placed in groups I-III and section 1 (Table 1) by Briggs (1953b). These groups consisted of a number of strains received as Lactobacillus acidophilus or L. bulgaricus, a few of $L$. delbrueckii, L. helveticus, L. lactis and L. jugurt and other unnamed and freshly isolated strains. For comparison, a few further cultures of named strains of lactobacilli were examined by microtests.

The details of the maintenance procedure and the media used for the routine culture and storage of these organisms were given by Briggs $(1953 b)$. The media used in the tests reported here are given under the test headings. The inoculum for all tests, except the microtests, was $0.05 \mathrm{ml}$. of a vigorously growing culture, usually after incubation for $18 \mathrm{hr}$. at 37 or $45^{\circ}$, or after $42 \mathrm{hr}$. at $30^{\circ}$. All tests were repeated at a later stage to confirm that the results were reproducible.

In all these tests a good medium for growth is essential. It is also imperative that the culture used for the inoculum is growing well. This was demonstrated 
clearly at one stage in this work: two cultures, which were difficult to classify but were thought to be Lactobacillus acidophilus or $L$. bulgaricus, gave fermentation results very different from other strains of these species. When the cultures were re-tested by the methods of Briggs (1953 $b$ ), but with heavier inocula of actively growing cultures, they were found to be strains of the heterofermentative species $L$. fermenti. It has also been found that tomato juice used in the basal medium gives much better growth after a period of storage at $4^{\circ}$ instead of at room temperature.

Table 1. Physiological classification of the lactobacilli*

\begin{tabular}{|c|c|c|c|c|c|c|c|c|c|}
\hline & \multirow{3}{*}{$\begin{array}{c}\text { Gas } \\
\text { from } \\
\text { glucose }\end{array}$} & \multirow{3}{*}{$\begin{array}{c}\mathrm{NH}_{3} \\
\text { from } \\
\text { arginine }\end{array}$} & \multicolumn{2}{|c|}{ Survival at } & \multirow{2}{*}{\multicolumn{3}{|c|}{ Growth at }} & \multirow{3}{*}{$\begin{array}{l}\text { Tolerance } \\
4 \% \mathrm{NaCl}\end{array}$} & \multirow{3}{*}{$\begin{array}{l}\text { Number } \\
\text { of strains }\end{array}$} \\
\hline & & & & & & & & & \\
\hline & & & $30 \mathrm{~min}$. & $30 \mathrm{~min}$. & $15^{\circ}$ & $45^{\circ}$ & $48^{\circ}$ & & \\
\hline Group I & - & - & - & - & - & + & - & - & $\mathbf{3 4}$ \\
\hline Group II & - & - & - & - & - & - & - & - & 16 \\
\hline Group III & - & - & + & - & - & + & - & - & 20 \\
\hline Group IV & - & - & - & - & + & + & + & + & 25 \\
\hline Group V & - & - & + & + & - & + & + & - & 82 \\
\hline Group VI & - & - & - & - & + & - & - & \pm & 127 \\
\hline Group VII & + & + & - & - & + & - & - & \pm & 55 \\
\hline Group VIII & + & + & - & - & - & + & - & \pm & 31 \\
\hline Section 1 & - & - & \pm & - & - & + & + & - & 24 \\
\hline Section 2 & - & - \pm & 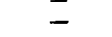 & - & - & + & - & + & 11 \\
\hline Section 3 & - & - & - & - & + & + & - & + & 12 \\
\hline Section 4 & & & Misc & ellaneous & react & & & & 15 \\
\hline
\end{tabular}

Microtests. These included the methyl red test, hydrolysis of starch, utilization of citrate, production of indole, production of acetoin, reduction of nitrate and production of $\mathrm{H}_{2} \mathrm{~S}$. The tests were carried out according to Clarke \& Cowan (1952) except that the cell suspensions were prepared as follows. Plates of tomato glucose agar were inoculated by spreading $0.5 \mathrm{ml}$. of $18 \mathrm{hr}$. cultures over the surface. The plates were incubated anaerobically; those cultures which grew at 37 and $45^{\circ}$ were incubated for $24 \mathrm{hr}$. and those which grew at $30^{\circ}$ for $48 \mathrm{hr}$. Growth was washed from the surface of the plates with sterile distilled water and the suspension centrifuged. The cells were then resuspended in distilled water, diluted to a standard opacity on an absorptiometer, recentrifuged and finally resuspended in one-tenth the volume of distilled water necessary to give the standard opacity. This concentrated suspension was used for the microtests.

Carbohydrate fermentation. The basic medium, consisting of Neopeptone $1.5 \%$, Tween $800.1 \%$, Yeastrel $0.6 \%$, and agar $0.15 \%$, had a final $\mathrm{pH}$ of $7 \cdot 0-7 \cdot 2$ and was sterilized by autoclaving at $15 \mathrm{lb}$. sq. in. for $20 \mathrm{~min}$. The indicator was $0.04 \%$ chlorophenol red. As the colour of an ethanolic solution was found to deteriorate when stored, the solid was dissolved in $1 \mathrm{ml}$. of ethanol and added to the medium immediately before tubing and sterilization.

Thirty 'carbohydrates' were used and $2 \%$ solutions, sterilized by Seitzfiltration, added to tubes of the basic medium to give a final concentration of 
$0 \cdot 2 \%$. Before inoculation the tubes were incubated for $24 \mathrm{hr}$. to check sterility. Inoculated tubes were incubated for 7 days and readings taken on the 1st, 2nd, 4th and 7th days.

Although conflicting results by earlier workers have thrown some doubt on the value of the fermentation tests, they have given clear-cut results in this work and confirm the findings of Rogosa et al. (1953). The basal medium supported good growth in the presence of added sugar, and a $2 \%$ inoculum was used. Since the inoculum was large, false positive results given by traces of acid in the inoculum had to be avoided by using an indicator with a low $\mathrm{pH}$ range; chlorophenol red ( $\mathrm{pH} 4 \cdot 8-6 \cdot 4)$ was selected.

Bile salt tolerance. Tomato glucose broth of the following compositiontomato juice $10 \%$, Neopeptone $1.5 \%$, glucose $2 \%$, sodium chloride $0.5 \%$, Tween $800 \cdot 1 \%$, Yeastrel $0.6 \%$, and soluble starch $0.05 \%$-was prepared, and to one-half of this basal medium $2 \%$ sodium tauroglycocholate was added, the other half serving as a control. Both the control and the bile medium were filled in $5 \mathrm{ml}$. amounts into $\frac{1}{4} \mathrm{oz}$. McCartney bottles and sterilized for $20 \mathrm{~min}$. at $15 \mathrm{lb}$. The inoculated bottles were incubated at $37^{\circ}$ and read after $24 \mathrm{hr}$.

$\mathrm{NaCl}$ tolerance. Tests for $\mathrm{NaCl}$ tolerance were carried out in exactly the same way as those for bile tolerance, except that $2 \%$ sodium chloride was substituted for sodium tauroglycocholate.

Starch tests. The most suitable medium for this test was found to be: Neopeptone $1.5 \%$, sodium chloride $0.5 \%$, Tween $800.1 \%$, Yeastrel $0.6 \%$, galactose $0.5 \%$, soluble starch $0.2 \%$ and agar $2 \%$. It was noticed that the presence of glucose diminished the hydrolysis of starch; the addition of a carbohydrate, however, greatly stimulated the growth of lactobacilli, and galactose, which was not found to affect starch hydrolysis, was added to the medium. The cultures were streaked on the surface of plates of this medium and incubated in an atmosphere of $90 \%$ hydrogen $+10 \%(\mathrm{v} / \mathrm{v})$ carbon dioxide at $37^{\circ}$ for 3 days. They were tested for hydrolysis of starch by flooding the plates with a solution of iodine in potassium iodide.

\section{RESULTS}

The results of these tests, with the exception of the microtests, allow the separation of 78 of the 94 organisms studied into three well-defined groups (Table 2). These are Lactobacillus acidophilus, L. bulgaricus, and a further group with reactions intermediate between the other two. It is also possible, using these tests in conjunction with those described by Briggs (1953b), to separate these organisms from others strains of lactobacilli. Of the remaining 16 strains, a further 5 were identified as $L$. lactis and 2 as $L$. fermenti; 9 were not classified, but it is, of course, possible that these belong to other species which have not yet been examined in detail.

The microtests were found to be unsuitable for classifying these organisms since the results were not reproducible. It is possible that failure was due to the fact that it is very difficult to prepare standard cell suspensions of lactobacilli, for the following reasons. The suspensions used in this work were of standard 
optical density, but, because of differences in size of the cells, they did not necessarily contain approximately equal numbers, nor were the proportions of dead and living cells constant. The cells for these tests are harvested and tested at a certain stage in their growth, unlike an inoculum in cultural tests, which continues to multiply during the test. Standard times and temperatures of incubation do not always produce suspensions which are identical, a slight variation in a batch of medium, for instance, may cause a slower rate of growth, and consequently give a suspension of different composition from that of a rapidly growing culture, although both have been incubated under identical conditions.

Table 2. Differentiating physiological characteristics of the three groups.

\begin{tabular}{|c|c|c|c|}
\hline & $\begin{array}{l}\text { Lactobacillus } \\
\text { acidophilus }\end{array}$ & Intermediate & $\begin{array}{c}\text { Lactobacillus } \\
\text { bulgaricus }\end{array}$ \\
\hline No. of strains in group & 29 & 15 & 34 \\
\hline \multicolumn{4}{|l|}{ Growth at } \\
\hline $45^{\circ}$ & $+(86 \%)^{*}$ & + & $+(85 \%)$ \\
\hline $48^{\circ}$ & - & $+(60 \%)$ & $-(76 \%)$ \\
\hline Tolerance of $2 \% \mathrm{NaCl}$ & + & + & - \\
\hline Tolerance of $2 \%$ Bile & $+(83 \%)$ & - & - \\
\hline Starch hydrolysis & $+(59 \%)$ & - & - \\
\hline \multicolumn{4}{|c|}{ Carbohydrate fermentations $\dagger$} \\
\hline Glucose & + & + & + \\
\hline Galactose & + & + & $+(85 \%)$ \\
\hline Lactose & + & + & $+(94 \%)$ \\
\hline Fructose & + & $+(73 \%)$ & $+(74 \%)$ \\
\hline Mannose & + & + & $+(91 \%)$ \\
\hline Dextrin & $+(83 \%)$ & + & - \\
\hline Maltose & $+(89 \%)$ & + & - \\
\hline Trehalose & $+(72 \%)$ & $-(60 \%)$ & - \\
\hline Amygdalin & + & - & - \\
\hline Cellobiose & + & - & - \\
\hline Salicin & + & - & - \\
\hline Sucrose & + & - & - \\
\hline Melibiose & $+(62 \%)$ & - & - \\
\hline Raffinose & $+(62 \%)$ & - & - \\
\hline Glycogen & $-(57 \%)$ & - & - \\
\hline
\end{tabular}

* The figures in parentheses indicate the percentage of strains giving the specified reaction.

$\dagger$ The following compounds were not fermented by any strains: adonitol, aesculin, arabinose, dulcitol, erythritol, glycerol, inositol, inulin, mannitol, melezitose, rhamnose, sorbitol, sorbose, starch and xylose.

\section{LACTOBACILLUS ACIDOPHILUS}

There were 29 organisms in this group: 15 strains named Lactobacillus acidophilus and 2 L. bulgaricus, 11 isolated from parts of the mammalian gut or from faeces and one from the mouth. It can be seen in Table 2 (footnote) that fifteen 'sugars' were not fermented by any strain, nine were fermented by all strains and the remaining six gave variable results. Melibiose and raffinose were fermented by $62 \%$ of the strains, and a strain positive to one was always positive to the other. It is of interest that those sugars which were not fermented by all strains were also those which showed differences in the results 
of duplicate tests of the same organism. The differences were, however, comparatively infrequent, occurring in only $1.3 \%$ of the tests.

Omitting those sugars giving variable results, four of the remainder, amygdalin, cellobiose, salicin and sucrose, which were fermented by all strains in this group and by none in the other two groups, can therefore be selected for purposes of differentiation.

No strains in this group grew in the presence of $4 \%$ bile salt but the majority tolerated $2 \% ; 5$ strains would not grow in either concentration. All strains grew in $2 \%$ sodium chloride.

The test for hydrolysis of starch did not give clear-cut results, and was therefore of little value.

\section{LACTOBACILLUS BULGARICUS}

This group contains 34 organisms: 18 strains named Lactobacillus bulgaricus and $2 L$. helveticus, 9 strains isolated from sour milk preparations or animals fed with these, 3 from présure and two were of unknown origin. Only glucose was fermented by all strains, although lactose, mannose and galactose were fermented by the majority. Fructose, which was unheated (since all sugars were sterilized by Seitz-filtration), was fermented by $74 \%$. No acid was produced from the remaining sugars, which included maltose and sucrose.

All strains in this group failed to grow in either $2 \%$ bile salt or $2 \%$ sodium chloride, and none of them hydrolysed starch.

\section{INTERMEDIATE GROUP}

In this group there are 15 organisms: 4 strains named Lactobacillus helveticus and 2 L. jugurt, 6 isolated from présure and 3 from milk. All these strains fermented glucose, galactose, lactose and mannose, in common with the first group of organisms and most strains of the second. They did not, however, ferment the four sugars, amygdalin, cellobiose, salicin and sucrose, fermented by $L$. acidophilus. Dextrin and maltose were fermented by all 15 strains; $\boldsymbol{L}$. bulgaricus strains and a few of the $L$. acidophilus strains were negative to these two sugars.

No strain in this group tolerated $2 \%$ bile salt, nor hydrolysed starch, but they all grew in $\mathbf{2} \%$ sodium chloride.

\section{DISCUSSION}

It has been shown that Lactobacillus acidophilus and $L$. bulgaricus are distinct species and using the test conditions described here, it is possible to distinguish clearly between them, and to define a further group with properties intermediate between the two. The bile salt and $\mathrm{NaCl}$ tolerance tests, which have proved of great value in the identification of the organisms in the work reported here, are not mentioned in Bergey's Manual (Breed et al. 1948) nor are three of the four sugars which gave clear results in the fermentation tests.

Other tests which were tried, but were later discarded, include the VogesProskauer test and a test for production of tyrosine decarboxylase, using the method described by Sharpe (1948). 


\section{Colonial appearance}

It was not possible to use colonial appearance as a distinguishing characteristic because of its variability. In the course of this work it was found that surface colonies of these organisms were not always similar. A plate prepared from one culture often gave a mixture of colonies, varying in shape and size, and standardization of techniques did not prevent this. Serological examination of different colonies (Sharpe, 1955) confirmed that they were identical with the original culture.

\section{Carbohydrate fermentation}

Rogosa et al. (1953) described strains of Lactobacillus acidophilus with properties similar to those described here; however, mainly on the basis of a weak or slow reaction in a single sugar, they recognized four variant types. In the present work the cultures of $\boldsymbol{L}$. acidophilus showing variable reactions to one or two sugars were classified together in a single group, and the cultures put into the intermediate group vary in a number of characteristics from the other two groups. It was felt that a number of similar characteristics would give groups that could be clearly and easily recognized with less risk of error than would occur when a single characteristic was used.

It is stated in Bergey's Manual that Lactobacillus bulgaricus produces acid from glucose, lactose and galactose, but that there are conflicting opinions on the value of fructose, maltose and sucrose. Rahe (1918), for instance, used only maltose for the classification since some of his strains fermented sucrose. Kulp \& Rettger (1924) found that $L$. bulgaricus would ferment heated fructose but not the unheated sugar. In the work described here, however, two-thirds of the strains of $L$. bulgaricus produced acid from unheated fructose, but none fermented maltose or sucrose. These findings are similar to those of Sherman \& Hodge (1940). These authors also say that some old cultures of $\boldsymbol{L}$. bulgaricus acquire the ability to ferment maltose and sucrose after prolonged laboratory culture. None of the strains in our collection possesses this property, although some of them have been cultivated in the laboratory for many years.

\section{Serology}

Serological work on these organisms (Sharpe, 1955) has confirmed the identity of some of the strains. Twenty-three of the 34 Lactobacillus bulgaricus strains have been found to belong to the same serological group, 6 have not yet been identified serologically and the remaining 5 belong serologically to L. lactis. Other strains of $L$. lactis which have been examined show very different sugar fermentations from the organisms grouped as L. bulgaricus. Further light may be thrown on this problem when the remaining cultures of L. lactis in our collection are examined in detail. Eleven of the 15 strains in the intermediate group are also serologically related. Sera have not yet been prepared for the L. acidophilus group. 


\section{Growth temperatures}

In describing groups I-III of lactobacilli, Briggs (1953 $b$ ) stated that although they were clearly separated from the remaining groups, they were not readily distinguishable from each other (Table 1), an opinion confirmed by this work. The strains comprising groups I-III were found, in the work reported here, to be distributed differently between Lactobacillus acidophilus, L. bulgaricus and the intermediate group (Table 3), indicating that growth

Table 3. Distribution of strains in Briggs groups $I, I I$ and III between Lactobacillus acidophilus and $\mathrm{L}$. bulgaricus

\begin{tabular}{lccccc} 
& \multicolumn{5}{c}{ Intermediate } \\
Group I* & No. of strains & L. acidophilus & strains & L. bulgaricus & Unclassified \\
Group II & $\mathbf{3 4}$ & $\mathbf{2 5}$ & $\mathbf{0}$ & 6 & $\mathbf{3}$ \\
Group III & $\mathbf{1 6}$ & 4 & 0 & 8 & 4 \\
Section 1 & $\mathbf{2 0}$ & 0 & 6 & 12 & 2 \\
& \multicolumn{2}{r}{ * Data from Briggs $(1953 a)}$.
\end{tabular}

temperatures cannot be used to distinguish between these organisms, in spite of their value in separating them from other groups. Growth temperatures have been used for classification by a number of workers, including Tittsler, Geib \& Rogosa (1947), Curran et al. (1933) and Sherman \& Hodge (1940), with varying degrees of success, and it seems apparent that whereas they are not always reliable as the sole means of identification, they are useful as supplementary tests. Much of the conflicting evidence is due to the use of different methods. It is obvious, for instance, that a large inoculum of an active culture is more likely to grow at a higher temperature than a small inoculum of a weak culture, since the chances of selecting resistant cells are greater. It is also more likely that a culture will grow at a temperature nearer to its maximum in an incubator than in an accurately controlled water-bath, since frequent opening of the incubator can cause considerable lowering of the temperature for varying periods of time. Most cultures used in this work had been found by Briggs $(\mathbf{1 9 5 3} b)$ to grow at $45^{\circ}$ and of those that at first failed, some have now been found to grow, if a larger inoculum is used. None of the cultures of L. acidophilus grew at $48^{\circ}$ but some of those in the intermediate and L. bulgaricus groups grew at that temperature.

The names Lactobacillus acidophilus and $L$. bulgaricus have been used for two of the species described in this paper. Each group includes a number of named cultures of the species and representative strains from the National Collection of Type Cultures, and the American Type Culture Collection. The results described here supplement the original descriptions (Luerssen \& Kuhn, 1908; Moro, 1900) rather than differ from them, except for the optimum growth temperature of $L$. bulgaricus. This was stated by the original authors (Luerssen \& Kuhn, 1908) to be from 45 to $50^{\circ}$. It is difficult to define the optimum temperature, which may, of course, vary with the medium used, but the cultures described here as $L$. bulgaricus had in most cases a maximum 
temperature between 45 and $48^{\circ}$. It is probable that the difference lies in the different techniques used for ascertaining growth temperatures, and also in the length of time the cultures have been cultivated in the laboratory. Sherman \& Hodge (1936) found that freshly isolated cultures of $\boldsymbol{L}$. bulgaricus would grow vigorously at $55^{\circ}$ but after a year the same culture would not grow at $50^{\circ}$.

According to the International Code of Nomenclature (Buchanan, St JohnBrooks \& Breed, 1948) it is not permissible to exclude the original types when re-defining a group, but in this case the original type strains are not in existence, and amplification of the definition, to indicate that older cultures may have a lower maximum temperature than fresh isolates, is all that is required. This seems a more rational solution than to rename all the cultures of Lactobacillus bulgaricus on the grounds that their lower maximum growth temperature excludes them from that group. It is also in accordance with Rule $\mathbf{1 7}$ of the Code. Descriptions of L. acidophilus and L. bulgaricus in Bergey's Manual may now be supplemented by the following:

\section{LACTOBACILLUS ACIDOPHILUS}

No growth at $20^{\circ}$ or below, and no growth at $48^{\circ}$ and above. Grows well at $37^{\circ}$.

Distinctive characters: Homofermentative. Grows in acid media. Ferments amygdalin, cellobiose, salicin and sucrose. Will not grow at $48^{\circ}$. Growth in $2 \%$ but not in $4 \% \mathrm{NaCl}$ or bile salt.

\section{LACTOBACILLUS BULGARICUS}

No growth at $20^{\circ}$ or below ; maximum temperature for growth of fresh isolates may be $50^{\circ}$ or above, but for older cultures it is generally between $45^{\circ}$ and $50^{\circ}$.

Distinctive characters: Homofermentative. Does not ferment amygdalin, cellobiose, salicin or sucrose. Growth at $48^{\circ}$ variable. Will not grow in $2 \% \mathrm{NaCl}$ or bile salt.

The group with properties intermediate to those of Lactobacillus acidophilus and $L$. bulgaricus has not been named. There are two alternatives, the first that it is a variety of $L$. bulgaricus, since it resembles that species more closely than $L$. acidophilus, and the second that it is a distinct species. It is felt that further and more detailed examination of these cultures is required to determine whether the characteristics in which these strains differ from L. bulgaricus are sufficient to define a separate species.

I wish to thank Dr A. T. R. Mattick for his helpful suggestions and advice during this work, and Miss P. Burrows for her technical assistance.

\section{REFERENCES}

Breed, R. G., Murray, E. G. D. \& Hitchens, A. P. (1948). Bergey's Manual of Determinative Bacteriology, 6th ed. p. 349. London: Baillière, Tindall and Cox.

Briggs, M. $(1953 a)$. The taxonomy and characterization of the lactobacilli. Thesis. University of Reading.

BrigGS, M. (1953 $b$ ). The classification of lactobacilli by means of physiological tests. J. gen. Microbiol. 9, 234.

Buchanan, R. E., St John-Brooks, R. \& Breed, R. S. (1948). International Bacteriological Code of Nomenclature. J. Bact. 55, 287. Reprinted 1949, J. gen. Microbiol. 3, 444.

Clarke, P. H. \& Cowav, S. T. (1952). Biochemical methods for bacteriology. J. gen. Microbiol. 6, 187. 
Curran, H. R., Rogers, L. A. \& Whittier, E. O. (1983). The distinguishing characteristics of $L$. acidophilus. J. Bact. 25, 595 .

KULP, W. L. \& RETTGer, L. F. (1924). A comparative study of Lactobacillus acidophilus and Lactobacillus bulgaricus. J. Bact. 9, 357.

Luerssen, A. \& Kunn, M. (1908). Yoghurt, die bulgarische Sauermilch. Z Zbl. Bakt. (2. Abt.), 20, 234.

Metchnikoff, E. (1910). The Prolongation of Life, 2nd ed. London: Heinemann. Mono, E. (1900). UUber den Bacillus acidophilus. Jb. Kinderheilk, 61, 687.

Orla-Jensen, S. (1919). The Lactic Acid Bacteria. Copenhagen: Andr. Fred. Host and Son.

RAHE, A. H. (1918). The classification of aciduric bacteria. J. Bact. 3, 407.

Rettger, L. F. \& Cheplin, H. A. (1921). A Treatise on the Transformation of the Intestinal Flora, with special Reference to the Implantation of Bacillus acidophilus. New Haven: Yale University Press.

Rogosa, M., Wiseman, R. F., Mitchell, J. A., Disraely, M. N. \& Beaman, J. A. (1953). Species differentiation of oral lactobacilli. J. Bact. 65, 681.

Sharpe, M. E. (1948). Some biochemical characteristics of group D streptococei isolated from infants' faeces. Proc. Soc. appl. Bact. 11, 13.

ShaRPE, M. E. (1955). A serological classification of lactobacilli. J. gen. Microbiol. $12,107$.

Sherman, J. M. \& Hodge, H. M. (1936). The thermophilic and anaerobic nature of Lactobacillus bulgaricus. Science, 84, 208.

Sherman, J. M. \& Hodge, H. M. (1940). The value of certain tests in the differentiation of Lactobacillus bulgaricus from Lactobacillus acidophilus. J. Bact. 40, 11.

Trtesler, R. P., Geib, D. S. \& Rogosa, M. (1947). Taxonomy of the genus Lactobacillus, with special reference to correlations of differential characteristics. J. Bact. 54, 12. 